Conclusions Risk factors for prediabets are the same as those for T2DM, this findings suggest that common pathophisiogical basis underlies the two diseases.

\section{P2-393 CARDIOVASCULAR AND DIABETES RISK IN PERSONS WITH EARLY GLUCOSE METABOLISM IMPAIRMENTS}

doi:10.1136/jech.2011.142976l.23

T Dzebisashvili.* Moscow Regional Research Clinical Institute named by M.F.Vladimirskiy, Moscow, Russia

Aim To assess RR of type 2 diabetes (T2DM), overall and acute cardiovascular mortality and cardiovascular events in persons with impaired fasting glucose (IFG) and impaired glucose tolerance (IGT). Materials and Methods According to population based study among 2508 adults, 3-year risk of T2DM, overall and acute cardiovascular mortality and cardiovascular events (myocardial infarction and stroke, coronary heart disease) was estimated in people with IFG, IGT, IFG+IGT diagnosed in 2006 in comparison with normal glucose tolerance. RR and regression coefficient (B) was calculated. RR of T2DM, cardiovascular events was adjusted for age, sex, BMI, systolic blood pressure, smoking.

Results Highest percent of transformation to T2DM and adjusted RR of T2DM was in IFG+IGT (33.3\% and 11.2 [3.93-31.65], $\mathrm{p}<0.01)$. Lowest percent of transformation to T2D and RR of T2DM was in isolated IGT (10.3\% and 3.92 [1.11-13.90], $p=0.034)$. Adjusted RR of cardiovascular mortality was significantly 3.2-fold higher in IFG. IGT and newly diagnosed T2D had significantly 3.6-fold and 2.3-fold greater risk of overall mortality. RR of cardiovascular events was significantly increased 2.2-fold in IFG and 2.7-fold in newly diagnosed T2D. There was not linear association between blood glucose levels and cardiovascular mortality risk $(p=0.095)$ in contrast to the continuous linear relationship observed between blood glucose levels and coronary heart disease risk $B=0.273$ ( $p=0.001)$.

Conclusion 3-year risk of developing T2DM is not equal at different early glucose metabolism impairments. IFG increased 3year risk of acute cardiovascular mortality. These may provide insights, that hyperinsulinemia influence on acute cardiovascular mortality risk.

\section{P2-394 INFECTION CONTROL PRACTICES AT THE MINISTRY OF HEALTH DENTAL CLINICS IN ALEXANDRIA}

\section{doi:10.1136/jech.2011.142976l.24}

${ }^{1} \mathrm{~N}$ El-Nimr, ${ }^{*}{ }^{1} \mathrm{~A}$ Hasab, ${ }^{1} \mathrm{M}$ Arafa, ${ }^{2} \mathrm{M}$ A Salam, ${ }^{1} \mathrm{M}$ Shama. ${ }^{1}$ High Institute of Public Health, Alexandria, Egypt; ${ }^{2}$ Faculty of Dentistry, Alexandria, Egypt

Introduction Infection control (IC) is a priority consideration in dentistry. Dental procedures are done in a septic environment posing significant hazards to dentists and patients. The Aim was to assess the dentists' IC practices at the $\mathrm{MOH}$ dental clinics.

Methods A cross-sectional design was used and the researcher visited 29 dental care facilities, selected randomly from Alexandria seven health districts. Two data collection tools included: 1- An IC checklist to study the six IC practices. Two data collection tools included: 1- An observational IC checklist to study the six IC practices (232 observation for each practice). 2- Dental clinic checklist. The score percent was calculated for each observation.

Results Gloves and masks were the most commonly available personal protective equipment. Handwashing sinks were were not dedicated for handwashing. Dry heat ovens were the most commonly used sterilisation device. In only $4.3 \%$ and $32 \%$ of the observations, hand hygiene was performed before donning and after removal of gloves respectively. In $81 \%$, new pairs were used for each patient. In about one-quarter of the observations the dentists moved away from the dental unit and touched other environmental surfaces while donning gloves. In almost all observations, used needles were discarded in the sharp container. The one hand scoop technique was used for recapping needles in about 34\% of the observations. The environmental surfaces were neither wrapped nor disinfected in $78 \%$ of the observations.

Conclusion Most IC resources at the $\mathrm{MOH}$ dental clinics were available. The dentists' compliance with certain IC practices was found to be not satisfactory.

\section{P2-395 SOCIOECONOMIC INFLUENCES AT DIFFERENT LIFE STAGES ON SELF-RATED HEALTH IN GUANGZHOU, CHINA}

doi:10.1136/jech.2011.142976l.25

${ }^{1} \mathrm{~T}$ Elwell-Sutton, ${ }^{*}{ }^{2} \mathrm{C} O$ Jiang, ${ }^{2} \mathrm{~W} S$ Zhang, ${ }^{3} \mathrm{~K}$ K Cheng, ${ }^{1} \mathrm{~T} \mathrm{H}$ Lam, ${ }^{1} \mathrm{G}$ Leung, ${ }^{1} \mathrm{C}$ Mary Schooling. ${ }^{1}$ School of Public Health, Li Ka Shing Faculty of Medicine, The University of Hong Kong, Hong Kong; ${ }^{2}$ Guangzhou Occupational Diseases Prevention and Treatment Centre, Guangzhou Number 12 Hospital, Guangzhou, China; ${ }^{3}$ Department of Public Health and Epidemiology, University of Birmingham, Birmingham, UK

Introduction In long-term developed countries socioeconomic position across the life course is positively associated with health. We examined these associations in a developing country with a history of efforts to reorganise social hierarchy.

Methods Taking a life course perspective, we used multivariable logistic regression to analyse the association of socioeconomic disadvantage at four life stages (measured by parental possessions, education, longest-held occupation and current household income) with self-rated health in 19203 Chinese adults aged $\geq 50$ years from the Guangzhou Biobank Cohort Study (2005-2008). Model comparisons were used to determine whether the number of exposures to disadvantage (accumulation of risk) was more important than the life stage of exposure (critical periods).

Results Among men and women, socioeconomic disadvantage in childhood and currently was associated with poor health, as was disadvantage in early adulthood for men. Adjusting for adult healthrelated behaviour (smoking, alcohol use and physical exercise) altered these associations very little.

Conclusion Associations between socioeconomic disadvantage and health in this Southern Chinese population were broadly similar to those found in Western countries in terms of the accumulation of disadvantage across the life course. However, there were also important differences. In particular, occupation (in both sexes) and education (in women) were not independently associated with adult health. This suggests that the mechanisms linking socioeconomic position to health in China may be different from those in Western populations.

\section{P2-396 KAP ON ANAEMIA RELATED ISSUES AMONG THE ADOLESCENT GIRLS IN RURAL BANGLADESH: ANALYSIS AFTER A COMMUNITY BASED INTERVENTION}

doi:10.1136/jech.2011.142976l.26

${ }^{1}$ D Farhana, ${ }^{*}$ 'S H Talukder, 'S H Khan, ${ }^{1}$ S Haque, ${ }^{2} \mathrm{Z}$ Mahmud. 'Eminence, Dhaka, Bangladesh; ${ }^{2} \mathrm{Ml}$, Dhaka, Bangladesh

Introduction In Bangladesh a large population, especially adolescent girls are vulnerable to Iron Deficiency Anaemia (IDA). Around 3.9 million adolescent girls are affected by IDA. Thus, Eminence in collaboration with Micronutrient Initiative (MI) had piloted a project in rural context to improve KAP among them and reduce prevalence of anaemia.

Methods Total 600 adolescent's girls aged 16-19 years from five unions in northern part of Bangladesh were interviewed and followup them after supplementing Iron Folate Tablets and behaviour 